\title{
INFLUENCE OF PH AND TEMPERATURE ON SURFACE-ACTIVE PROPERTIES OF SOME HUMIC ACIDS
}

\author{
El-Bayaa, A. A. and Asmaa Al-Amir \\ Faculty of Science, Al-Azhar University (Girls)
}

\begin{abstract}
In this study, surface tension measurements were used to study the surface-active properties of olive humic acid (HAO) and commercial humic acid (HA2). The study was performed under different conditions of concentration, temperature, $\mathrm{pH}$ and time. The measurements showed that the amphiphilic character of the humic acids solutes at the air/water interface lowers the surface tension $(\gamma)$ of the solution with an increasing concentrations and temperatures. The critical micelle concentration (CMC) values of both humic acids decreased with an increase in the temperature. Positive values of $\Delta S^{\circ}$ and $\Delta H^{\circ}$ suggested that, their adsorption at air/water interface, is endothermic and entropy driven. The surface tension-pH curve featured a minimum for both humic solutions, declining from higher and lower $\mathrm{pH}$ values.
\end{abstract}

Key words: Surface tension, Humic acids, pH, Entropy, Olive

\section{1-INTRODUCTION}

Surfactants, literally, are active at a surface and that includes any of the liquid/liquid, liquid/gas or liquid/solid systems, so that the subject is quite broad. The surface activity of a solute molecule arises primarily from the ability of the adsorbed solute molecule to alter the attractive interactions at the interface between two dissimilar molecules (air-water or oil-water). The properties of surfactant like conductivity, surface tension etc, change with the concentration above or below CMC as well as on the length of the hydrophobic tail

(Rehman et al. 2017). Humic acids exhibit surface-active properties due to the presence of both hydrophilic and hydrophobic moieties in their structure same as in surfactantes. The presence of the hydrophobic group in the bulk medium causes a distortion of the water structure which leads to an increase in the free energy of the system. This means that less work is needed to bring a surfactant molecule, than a water molecule, to the surface and therefore, the surfactant concentration at the surface increases over that in the bulk, a phenomenon which is termed surface activity. The presence of a hydrophilic group and a hydrophobic group causes the surfactant to preferentially adsorb at water-gas interface. The surfactant tails have lower surface tension, $\gamma(\mathrm{mN} / \mathrm{m})$, than water and thus the surface tension of the water-gas interface is lowered. The magnitude of the reduction in depends on the type and the number of molecules adsorbed at the interface, which is a function of surfactant bulk concentration. The hydrophobic end is usually a carbon chain of roughly 8 to 20 carbon atoms, when the hydrophilic head contains a functional group (eg. $-\mathrm{COO}-$ or $-\mathrm{SO}_{3}$ ).

Their importance in agriculture field because, of its significant improvement of the soil's physical properties and moisture conditions, due to their surface activites, which leads to higher soil quality and productivity (Zhang and He, 2004), (Madejon et al., 2001, Kerek et al., 2003, Arancon et al., 2004, and Eladi et al., 2005).

Other industrial applications can be mentioned: as an ion exchanger, as a source of synthetic hydrocarbons and fuel oils (Duncan et al. 1981), in food- processing or to enhance the extraction of uranium from its ores (Schmeide et al. 2000). In aqueous solution surfactant molecules starts to aggregate and form micelle in concentration called ascritical micelle concentration (CMC) and is one of the most important physical property of the surfactants .The ability to influence the surface 
tension of humic acid solutions depends on the origin of the humic acids. (Klavins and Purmalis, 2013). The effect of $\mathrm{pH}$ onthe surface activity of HA has been reported (Terashima M. et al. 2004). The resultsindicate that both the surface activity and the aggregation are enhanced at low $\mathrm{pH}$.The determination of thermodynamic parameters of surfactant formation in aqueous solutions is more important, because they quantify the relative importance of hydrophobic interactions, surfactant-water contact and (for ionic surfactants) head-group repulsion. These parameters can be derived from the temperature dependence (Tennouga et al., 2015).

Therefore in the present study, we evaluated the ability of olive humic acid (HAO) and commercial humic acid (HA2) to influence surface tension. The surfactant effects of these materials were evaluated under different concentrations, $\mathrm{pH}$, temperatures and times.

\section{MATERIAL AND METHODS}

\subsection{Studied humic acids samples.}

\subsubsection{Commercial humic acids}

Commercial humic acids (HA2) were imported from china. It has $95 \%$ potassium humate appearance crystal granules of size 0.5 $-1.5 \mathrm{~mm}$, water solubility $12 \% \max , \mathrm{pH}=9$ 10.

\subsubsection{Olive cake compost}

The solid olive waste was derived from olive oil Alkttan factory in Cairo. The waste was composted without supporting with any plant residues, or additives, under uncontrolled atmospheric conditions. The extraction and purification of humic acids, (HAO) was conducted after stored about 2 years (Badr M.H. and Al-Amir. A, 2017) .

\subsection{Extraction of humic acids}

Extraction and purification of humic acids was carried out according to IHSS (International Humic Substances Society) with slight modifications( Swift, 1996), (Badr et al., 2005). The soil samples were equilibrated with
$(0.1 \mathrm{~N}) \mathrm{HCl}(10 \mathrm{ml} / \mathrm{g}$ dry weight soil) at room temperature for 24 hours and the supernatant was separated by decantation. $\mathrm{NaOH}(0.1 \mathrm{~N})$ was added to give the final extraction ratio. The extraction was carried out in plastic bottles. The air in the bottles was displaced by the extracting solution itself and shaked from time to time for 24 hours. After decantation and filtration $\mathrm{HCl}$ added to precipitate humic acid at $\mathrm{pH}(1-2)$. The precipitates were left 24 hours for coagulation and the crude humic acid olive was separated by decantation, filtration and centrifugation.

\subsection{Purification of humic acids}

Both humic acids (olive, commercial) were purified according to (Bader, 2003). Humic acids were dissolved under $\mathrm{N}_{2}$ gas in minimum volume of solution prepared by adding $\mathrm{KCl}$ solution to $(0.1 \mathrm{~N}) \mathrm{KOH}$ to attain a concentration of $(0.3 \mathrm{M})$ KI. The solution centrifuged at the highest speed for 15 mins. to remove the suspended solids. Then reprecipitated by acidification and left for coagulation for about 12 hours. The suspended in $(0.1 \mathrm{M}) \mathrm{HCl} /(0.3 \mathrm{M}) \mathrm{HF}$ solution in plastic bottles and shacked over night at room temperature (this step repeated three time) after separation the precipitate is dissolved in 0.05 $\mathrm{NNaOH}$ under $\mathrm{N}_{2}$ gas then adjusted to $\mathrm{pH}(1-2)$ by $1.0 \mathrm{~N} \mathrm{HCl}$ and left for $12 \mathrm{hrs}$.

The precipitate then washed by distilled water till negative chloride test and freezed. After freezing the gelatinous precipitate of humic acids changed into particles and collected after further washing them dried in oven at $40^{\circ} \mathrm{C}$ for three days. Finally, the purified humic acids were kept in desiccator.

2.4. Humicacid structure: The structure of humic acid is given in Fig.(1) and the function groups are represented in Table (1)

Both humic acids (HAO, HAC) have important groups that affect on their surface activity, such as $(\mathrm{COOH}, \mathrm{OH})$. showed in table (1). 


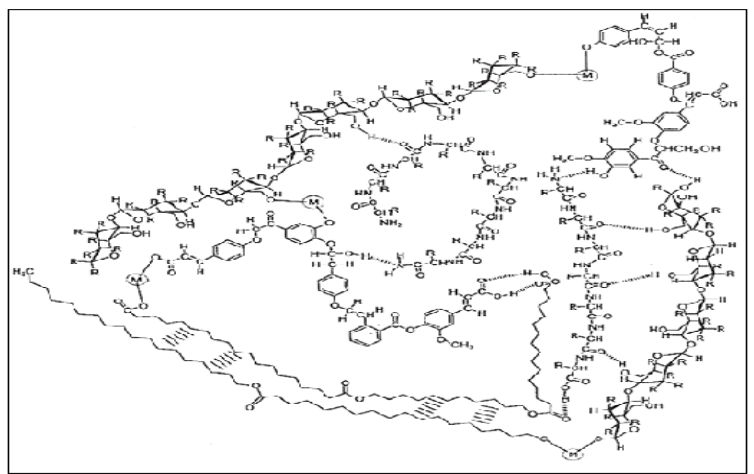

Fig. (1) Recent model structure of humic acid according to Simpson et al. , (2002).

Table (1) The acidity, carboxyl, and phenolic hydroxyl groups contents, of the studied humic acids.

\begin{tabular}{|c|c|c|c|c|}
\hline HA & $\begin{array}{c}\text { Total } \\
\text { acidity } \\
\text { meq/g }\end{array}$ & $\begin{array}{c}\text { COO } \\
\text { H } \\
\text { meq/g }\end{array}$ & $\begin{array}{c}\text { Acidic } \\
\text { OH meq/g }\end{array}$ & $\begin{array}{c}\text { Acidit } \\
\text { ratio }\end{array}$ \\
\hline HA2 & 12.55 & 4.39 & 8.17 & 0.54 \\
\hline $\begin{array}{c}\text { HA } \\
\text { O }\end{array}$ & 8.57 & 2.37 & 6.19 & 0.38 \\
\hline
\end{tabular}

\subsection{Solutions preparation}

Stock solution of concentration $(1000 \mathrm{mg} / \mathrm{L})$ for each humic acids isolated from olive (HAO) and commercial humic (HA2) were prepared in doubled distilled water by the aid of (0.1) $\mathrm{N}$ sodium hydroxide. Other concentrations were prepared from stock solution by dilution.

\subsection{Surface Tension Measurements}

The surface tension of aqueous solutions of humic acids isolated from olive (HAO) and commercial humic (HA2) were measured using stalagmometry, operating on the principle of a falling drop under controlled environmental conditions $\left(25 \pm 1 \mathrm{C}^{\circ}\right)$.

The accuracy of instrument was checked by frequent determination of the surface tension of water. Each experiment was repeated three or more times. Measurement of several solutions was repeated after a few days, and the results were found to be with the uncertainty limit reported.

\subsubsection{Effect of different humic acid concentrations on surface tension at different temperature}

The equilibrium surface tension of the olive humic acid (HAO) and commercial humic acid(HA2) in distilled water were investigated by determining the surface tension solutions at different concentrations ranging from 50 to 700 $\mathrm{mg} / \mathrm{L}$. These concentrations ranged from very low values to values at which the surface tension did not change any more. Surface tension with varying bulk concentrations were determined at different temperatures 25, 35 and $45^{\circ} \mathrm{C}$.

For any new measurement, the humic solution was first equilibrated at lowest temperature and then surface tension, $\gamma$ was measured after two hours. Then the temperature was raised and the solution was also equilibrated again. After getting the surface tension data for all dilutions for each humic solution at a given temperature. The plots of surface tension $(\gamma)$ versus $\log C$ (where $C$ is the concentration of humic the in $\mathrm{mg} / \mathrm{L}$ ) at each temperature, were used for the estimation of the critical micelle concentration (CMC) of the humic acids. The CMC, was assigned to the concentration at and above which the surface tension remains constant.

\subsubsection{Effect of $\mathbf{p H}$ solution on surface tension}

In order to evaluate the effect of $\mathrm{pH}$ on surface tension, solutions were prepared containing $500 \mathrm{mg} / \mathrm{L}$ of HA dissolved in $0.1 \mathrm{M}$ $\mathrm{NaOH} ; \mathrm{pH}$ values were adjusted between 9 and 2 with $0.1 \mathrm{M} \mathrm{HCl}$.

\section{RESULTS and DISCUSSION}

3.1.Interpretation of surface tension vs. concentration curve and determination of critical micelle concentration (CMC)

Studies on interfacial properties of olive humic acid (HAO) and commercial humic acid (HA2) with varying bulk concentrations ranging from 50 to $700 \mathrm{mg} /$ Lat temperatures 25,35 and $45^{\circ} \mathrm{C}$ were studied. 
Fig.(1) shows the effect of progressively increasing concentrations on the surface tension of solution. All plots of surface tension $(\gamma)$ versus logarithm of $\mathrm{HAO}$ and HA2 concentration $(\log C)$ for aqueous solutions show familiar surfactant behaviour. That is, by increasing humic acid amount in solution led to decrease in surface tension up to a specific concentration and beyond which no prominent decrease in surface tension was observed. Because of their chemical structure, HA have characteristics that promote their accumulation at the air-water interface, which causes a decrease in the surface tension (Quadri et al. 2008).

The $\mathrm{y}$-lnC plots for HAO and HA2 Fig.(2) are mostly sigmoid in nature. There are three distinct regions in this plot: (1) a decrease in surface tension as the HAO and HA2 concentration increases; (2) a point where the surface tension decrease abruptly stops and the surface tension becomes constant; this point is CMC and (3) a constant surface tension observed with further increase of concentration.

These regions are interpreted as follows. In region(1), the increasing humic concentrations progressively disrupt more and more of the interactions between water molecules. Additionally adsorption of HAO and HA2 at air/water interface resulting in decreasing surface tension. The regular decrease in surface tension of solution with concentration continues till the completion of Gibbs monolayer (saturation point). After which there is no more prominent change in the surface tension, which means that further humic unimers go into the bulk of solution and these monomers associate in the form of selfassembled structure called micelle.

At the saturation point, surfactant monomers are hardly accommodated at the interface, and $Y$ remains constant (or unchanged). It can be physicochemically considered that the polar or ionic head groups of surfactants at the interface remain hydrated. With increasing concentration, the available area at the interface per solvated ion or group decreases by way of competitive accommodation causing their desolvation, which in turn adds more surfactant molecules at the interface leading to the increase in concentration of the surfactant molecules at the interface and hence to the decline in $\gamma$. In this way, the interface gets significantly populated at the beginning of the region (3) and begin to self-associate (form micelles) in the liquid phase (Patilet al., 2003).

Micelles are 3D structures containing at least around hundred monomers arranged such that their hydrophilic heads face the water, shielding the hydrophobic tails from being exposed to water (Myers, 1988). In this model, the amphiphilic HA molecules are considered to "aggregate" both intra- and intermolecularly. The former is made possible by the chain length and flexibility of the humic polymers, which allow them to fold and coil in a manner that directs hydrophilic (e.g. carboxy and hydroxy) groups outward and keeps more hydrophobic (e.g. hydrocarbon) moieties isolated in the center (Wandruszka, 2000).

After region (3) $\mathrm{Y}$ does not practically change with increasing surfactant which, most often, is found in reports defining it to be the point of CMC (critical micelle concentration i.e., the start of micelle formation). Further addition of surfactant in the solution results in the increased formation of micelles, which are not surface active, and remain in the bulk. In this condition, the surfactant monomers in solution are considered to be in thermodynamic equilibrium with the micelles in one hand and with the adsorbed molecules (or ions) at the interface on the other. Also, the micelles are taken to be in equilibrium with the adsorbed molecules (or ions) at the interface. Surface tension is a dependable and elegant method for the determination of CMC. The CMC is one of the most important physical parameters of surfactants. The properties of a surfactant vary markedly, when its concentration is higher or lower than its CMC, and the studies and industrial applications of a surfactant are always based on the value of its CMC.

Above the CMC increasing the surfactant concentration does not reduce the surface 
INFLUENCE OF PH AND TEMPERATURE ON SURFACE-ACTIVE ...

tension any more, since no more surfactant molecules can reside at the interface and they begin to aggregate to micelles. Once micelles are formed in solution, the bulk concentration of surfactant unimers (unaggregated molecules) remains constant, as a result of which the surface tension remains constant above the CMC (Hagenhoff et al., 2010) .

Graphically the values of CMC were obtained from the point of intersection of two straight lines in the surface tensionconcentration plots. One straight line was drawn where the surface tension drastically decreases with surfactant concentration and the other from the region where it becomes nearly constant. The humic acids under study indeed possess surfactant properties. The (CMC) were 500 and $550 \mathrm{mg} / \mathrm{L}$ at $25^{\circ} \mathrm{C}$ for olive humic acid (HAO) and commercial humic (HA2) respectively.

Ionics, micellization is affected by temperature as the hydrophobic and head group interactions change with temperature. Accordingly, critical micelle concentration (CMC) versus temperature studies has been performed to obtain information on these interactions. For each temperature, the CMC was determined by the intersection of the two slopes of each curve. The values of CMC for HAO were 500,475 and 450while for HA2 were 550,500 and 475 at temperature 25,35 and $45^{\circ} \mathrm{C}$ respectively. The CMC decreases as temperature increases Fig. (3), due to the smaller probability of hydrogen bond formation at higher temperatures. In other words, the increase in temperature causes the reduction in hydration of the hydrophilic group, which favour micellezation (Mahmood et al., 2013).Consequently, as increase in temperature, the micellization onset occurs at lower concentrations. On the other hand, dissolving the surfactant molecules in distilled water makes the hydrophobic group distorts the water structure. Additionally increase in temperature also causes an increase in the breakdown of the structured water surrounding the hydrophobic groups, which disfavors micellization.
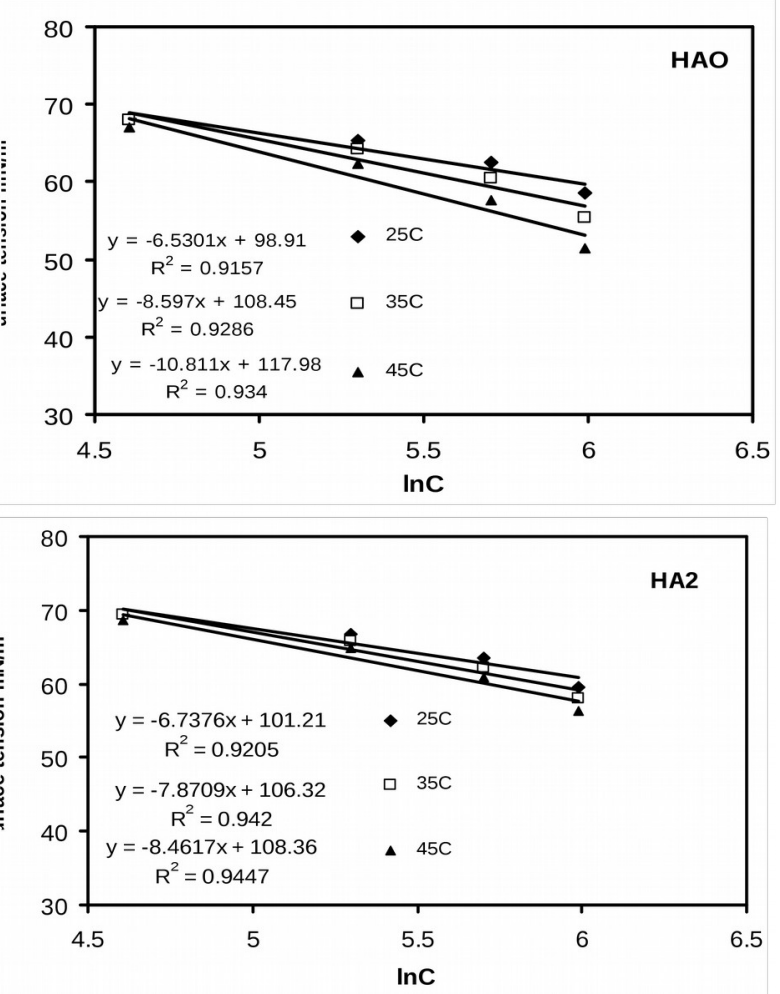

Fig. (2) The surface tension for humic acids olive(HAO) and commercial (HA2) at different concentration and temperature
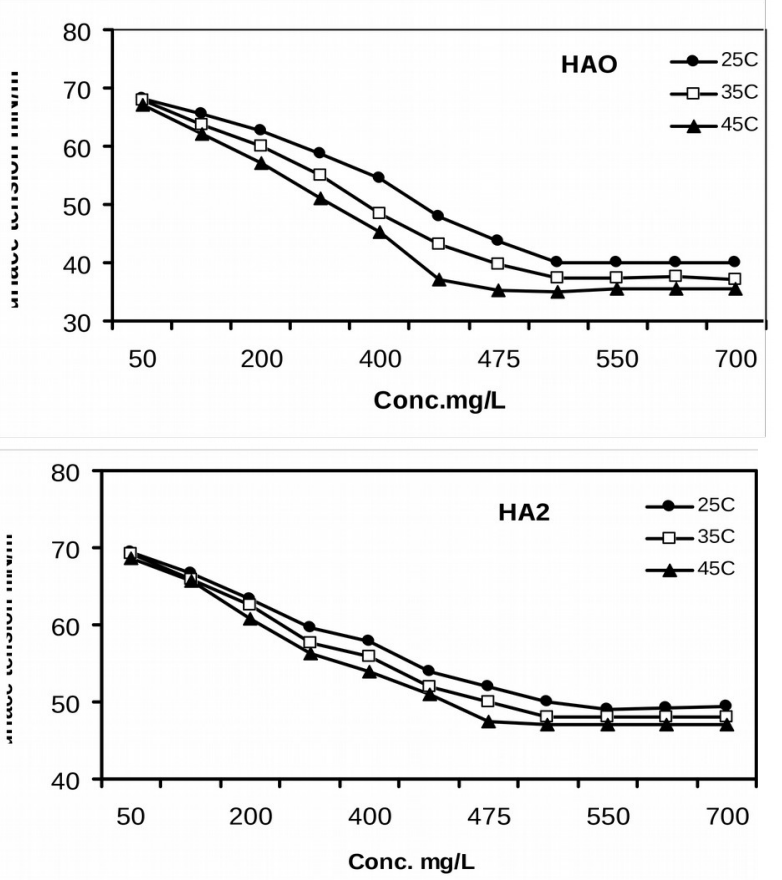

Fig. (3): CMC for humic acid (HAO) and humic acid (HA2) at different temperatures. 
Fig. (4): CMC for (HAO) compared to (HA2) at different temperatures.

\subsection{Thermodynamic parameters of adsorption at air/water interface}

The onset of adsorption characteristics of HAO and HA2 humic solutions at the air/water interface can be well studied using surface tension measurements. The evaluation of enthalpy change $\Delta \mathrm{H}$ by using the following Eq.

(1)(Ali K. et al., 2006)

$$
\Delta \mathrm{H}^{\circ}=\gamma-\mathrm{T}(\mathrm{d} \gamma / \mathrm{dT})_{\mathrm{C}}
$$

The relation between surface tension and temperature at different concentration Fig (5).All the lines of different temperatures are almost merged together which indicates a temperature independent nature of enthalpy in the present range of temperature. The data of the enthalpy $\left(\Delta \mathrm{H}^{\circ}\right)$ of surface formation of $\mathrm{HAO}$ and HA2 solutions have been given in Table (2). $\Delta \mathrm{H}^{\circ}$ is positive which means that energy is required to break the bonds between humic head groups of the unimers and water in transferring them to the interface. (Rosen et al., 1982) predicted that, steric factor is also responsible for the positive value of $\Delta \mathrm{H}^{\circ}$. The
Enthalpy of surface formation increases with concentration linearly for humic solutions under study.

The entropy change of surface formation $\Delta \mathrm{S}^{0}$ was calculated by using the relation (2): (Ali K. et al., 2006).

$$
\Delta \mathrm{S}^{\mathrm{o}}=-(\mathrm{d} \gamma / \mathrm{dT})
$$

The entropy of surface formation generally increases with concentration for these solutions as shown for example for HAO and HA2 in Table (2).The increase in the entropy with the concentration is quite obvious as higher the number of ions of solute present at the surface region, higher will be the disorder and thus higher value of entropy is expected. The $\Delta S^{0}$ values are positive for the studied system; it may be due to breaking of water structure when the humic hydrophobic chain transfers from bulk water to micellar core. Positive $\Delta \mathrm{S}^{0}$ means that freedom of humicunimers and hence movements occur at air/water interface. Positive values of $\Delta \mathrm{S}^{\circ}$ and $\Delta \mathrm{H}^{\circ}$ suggest that adsorption at air/water interface is endothermic and entropy driven.

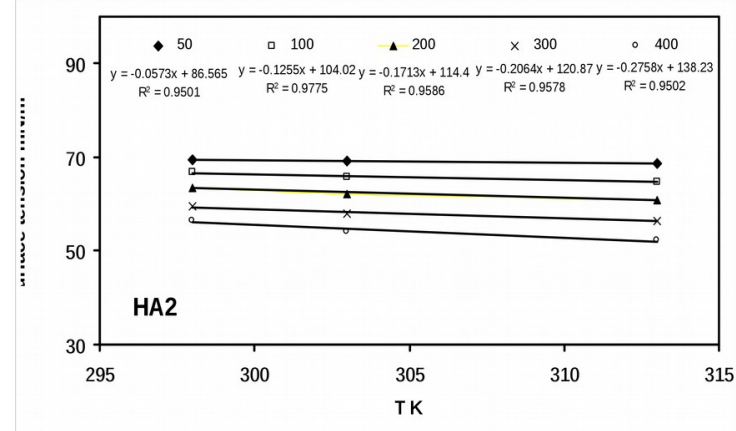

Fig. (5): The relation between surface tension and temperature for(HA2) at different concentration.

Table (2): The thermodynamic parameters at different concentrations and

\begin{tabular}{|c|c|c|c|c|c|c|c|c|}
\hline \multicolumn{4}{|c|}{ HA2 } & \multicolumn{4}{|c|}{ HAO } & \multirow{3}{*}{$\begin{array}{l}\text { Conc. } \\
\text { mg/L }\end{array}$} \\
\hline \multicolumn{3}{|c|}{$\begin{array}{c}\Delta \mathbf{H}^{\mathbf{0}} \\
\mathrm{mN} / \mathrm{m}\end{array}$} & \multirow{2}{*}{$\begin{array}{l}\Delta S^{0} 10^{6} \\
\mathrm{mN} / \mathrm{mk}\end{array}$} & \multicolumn{3}{|c|}{$\begin{array}{c}\Delta \mathbf{H}^{\mathbf{0}} \\
\mathrm{mN} / \mathbf{m}\end{array}$} & \multirow{2}{*}{$\begin{array}{l}\Delta S^{0} 10^{6} \\
\mathrm{mN} / \mathrm{mk}\end{array}$} & \\
\hline $45 C^{0}$ & $35 C^{0}$ & $25 C^{0}$ & & $45 C^{0}$ & $35 C^{0}$ & $25 C^{0}$ & & \\
\hline 85.518 & 85.442 & 86.480 & 57 & 87.973 & 88.142 & 88.930 & 70 & 50 \\
\hline 103.288 & 103.868 & 104.142 & 126 & 123.661 & 124.553 & 124.702 & 199 & 100 \\
\hline 113.238 & 113.610 & 115.115 & 173 & 159.580 & 160.217 & 160.672 & 329 & 200 \\
\hline 119.865 & 120.369 & 120.996 & 206 & 195.930 & 196.209 & 197.368 & 466 & 300 \\
\hline 137.477 & 137.746 & 138.656 & 276 & 205.322 & 206.129 & 208.807 & 519 & 400 \\
\hline
\end{tabular}
temperatures.

3.3. Effect of solution pH on surface tension $\mathrm{pH}$ have a major influence on the charge distribution and 
INFLUENCE OF PH AND TEMPERATURE ON SURFACE-ACTIVE ...

hydrophilicity of dissolved humic substances. The $\mathrm{pH}$ of a medium will control the magnitude of electrostatic charges which are imparted by the ionized molecules.

The structure of humic substances is characterized by the presence of numerous aromatic, carboxylic and phenolic functionalities, linked with alkyl moieties, imparting a measure of flexibility to the polymer chains. Generally, at high $\mathrm{pH}$, the positive charge at the solution interface decreases. In contrast, at a low $\mathrm{pH}$, the positive charge on the solution interface will increase. The Surface tension $(\gamma)$ profiles as a function of $\mathrm{pH}$ for olive humic acid (HAO) and commercial humic acid (HA2) Fig. (6). The surface tension-pH curve featured a minimum for both solutions, declining steeply from higher and lower $\mathrm{pH}$ values .The results indicate the measured surface tension was greatest at high $\mathrm{pH}$, since the humic solute was highly anionic (and therefore very hydrophilic) under those conditions. The samples exhibit $\mathrm{pH}$ dependence, relation between $\Delta \gamma$ and $\mathrm{pH}$ having a lower decrease in surface tension at $\mathrm{pH}$ values 4.0. Surface tension decreases from 60.16 to 38.43 and from 67.4 to $40.9 \mathrm{mN} / \mathrm{m}$ for $\mathrm{HAO}$ and HA2 respectively when $\mathrm{pH}$ is lowered from 9.43 to 4.0. This effect could be related to changes in the supramolecular structure of the humic acids, in such way that at this $\mathrm{pH}$ value, there is an arrangement of molecules that decrease surface tension.

The changes in surface tension as a function of $\mathrm{pH}$ can be related to the presence of functional groups, particularly $\mathrm{COOH}$. At high $\mathrm{pH}$, acid groups are fully ionized, and there is a strong repulsion between charged groups causing the molecule to expand (Tombácz , 1999). This phenomenon is also related to the buffer capacity of humic acids that has been related to the physisorption of protons and hydroxyl ions (Pertusatti, 2007). As well humic acids can form micelle structures that act as solubilizing agents for hydrophobic compounds.
The decrease in surface tension with decreasing $\mathrm{pH}$ reflects the gradual neutralization of humic acid-COO- ${ }^{-}$groups, which creates amphiphilic species that migrate to the air/water interface. This phenomenon is consistent with the functional group density distribution and molecule size, which allowed extensive $\mathrm{H}$-bonding with the aqueous solvent throughout the experimented $\mathrm{pH}$ range (Engebretson and Wandruszka, 1998). It can be observed that around $\mathrm{pH}=4, \mathrm{HA}$ are especially effective in forming amphiphilic molecules. The Surface tension increased again at low $\mathrm{pH}$, where continued neutralization produced more hydrophobic molecules with low surface activity.

The lowest surface tensions were obtained in humic containing more aliphatic groups and fewer carboxylic groups (HAO) , facilitating their neutralization and leading to rearrangement of hydrophobic and hydrophilic groups for micellar formation of the molecule. These results indicate that the HAO have a strong amphiphilic character compared with HA2 .

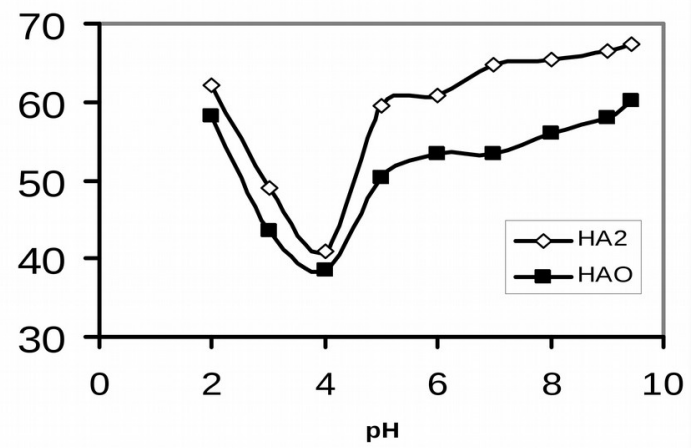

Fig. (6): Variation of the surface tension of (HAO), (HAC) with pH.

\subsection{Dynamic surface tension measurements}

The dynamic surface tension is the surface tension before equilibrium conditions are reached and depends on the age of the interface. The dynamic surface tension is an important property, which allows obtaining kinetic information of the surfactant adsorption at the interface. Time variations of surface tensions of aqueous HAO and HA2 solutions at $25 \mathrm{C}^{\circ}$, were investigated. When a fresh interface 
is created $(t=0)$, the interface consists only of water molecules and $\gamma=\gamma$ pure solvent (for water, $72 \mathrm{mN} / \mathrm{m}$ ).

Dynamic surface tension curves for HAO and HA2 solutions are displayed in Fig. (7). It is seen that the surface tension of fresh surfaces decreases with time to an equilibrium value because of the diffusion of surfactants from the bulk solution to the surface, and to their dynamic interaction on the surface, as expected. As well the HAO and HA2 tails have lower surface tension, $(\mathrm{mN} / \mathrm{m})$, than water and thus the surface tension of the water-gas interface is lowered. The decrease continued to equilibrium, at this time, the surface tension was 41.200 and $49.990 \mathrm{mN} / \mathrm{m}$ for the HAO and HA2 respectively, after this, change was not observed. The decrease in surface tension for the HAO and HA2 solutions continued until about 80 minutes. After this, change was not observed .The reduction depends on the type and the number of molecules adsorbed at the interface, which is a function of surfactant bulk concentration.

The change of $\gamma$ with time was fit to a second-order exponential decay. Second-order exponential decay was found to provide the best fit for all the data sets. This may indicate that at least two time dependent processes are involved in establishment of equilibrium: (i) diffusion of molecules to the surface, and (ii) conformational rearrangement of molecules at

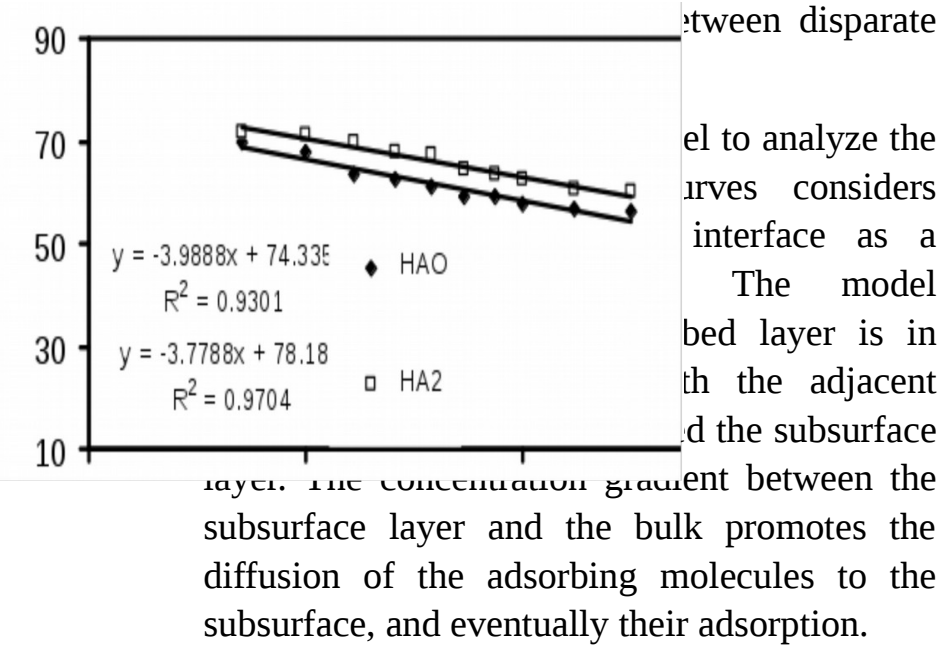

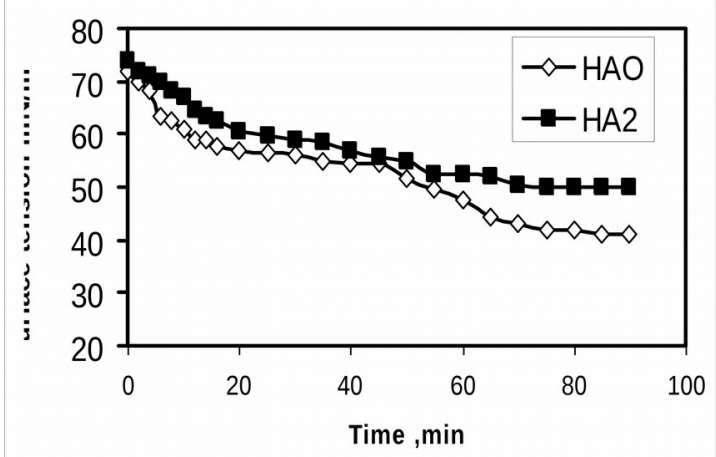

Fig. (7): Change in surface tension value as a function of time for (HAO) and (HAC).

For the diffusion-controlled adsorption mechanism a linear dependence of $\gamma$ on $\sqrt{ } t$ is expected (Thiam et al., 2012) which is represented by the relation (3)

$$
\left[\frac{d \gamma}{d \sqrt{t}}\right]=-2 R T c_{0} \sqrt{\frac{D}{\pi}}
$$

where $\gamma(\mathrm{t})$ denotes the dynamic interfacial tension, $\mathrm{R}$ the gas constant, $\mathrm{T}$ the absolute temperature, $\mathrm{C}_{0}$ the humic bulk concentration and D the diffusion coefficient. Fig.(8) shows the dynamic interfacial tension as a function of $\sqrt{t}$ for HAO and HA2 solutions. The linear relationship between $\gamma_{(t)}$ and $t$ is valid only for time periods of $t>100 \mathrm{~s}$ analysis of the curves for HAO and HA2 solutions indicated that a remarkable additional surface tension depression was experienced with long times. The calculated diffusion coefficients are $2.520 \times 10^{-10}$ and $2.261 \times 10^{-10}$ for HAO and HA2 respectively.

\section{$\sqrt{\mathbf{t}} \min$.}

Fig. (8): The dynamic interfacial tension as a function of $\sqrt{ } t$ for $H A O$ and $H A 2$ solutions.

\section{CONCLUSIONS}


INFLUENCE OF PH AND TEMPERATURE ON SURFACE-ACTIVE ...

HAO and HA2humic acids show typical polyelectrolytic properties and their conformation in the solution depends on $\mathrm{pH}$, aging time, and concentration. Their solutions are able to reduce surface tension in an aqueous solution and can form micelle-like aggregates above the critical micelle concentration (CMC). The decrease in surface tension with decreasing $\mathrm{pH}$ reflects the gradual neutralization of acidic sites, which created amphiphilic species that migrated to the surface. Positive values of $\Delta \mathrm{S}^{0}$ and $\Delta \mathrm{H}^{0}$ suggest that adsorption at air/water interface is endothermic and entropy driven. The research suggests that, HAO and HA2 surfactant would be beneficial in many of the material industry, agriculture and environment.

\section{REFERENCES}

Arancon, N.Q., Edwards, C.A., Bierman, P., Welch, C., Metzger, J.D., (2004). Influences of vermicomposts on field strawberries1.Effects on growth and yields. Bioresour. Technol. 93 (2), 145-153.

Ali K., ul-Haq A., Bilal S., Siddiqi S. (2006). Concentration and temperature dependence of surface parameters of some aqueous salt solutions, Colloids and Surfaces A: Physicochem. Eng. Aspects 272, 105-110. Badr M.H. and Asmaa Al-Amir.(2017) Testing the quality of some commercial humic acids used for agriculture in Egypt regarding with the regional soil and compost humic acids the $1^{\text {st }}$ international conference of national committee for pure and applied chemistry NCPAC17 Cairo, Egypt.

Badr, M.H., Abdel Gawad, L.I ,.and Shabon, M.H. (2005). Physiological effect of natural humic acid during pregnancy on fetuses and maternal alterations induced by irradiation in rats Isotop\&Rad.res.,37(3)749-772.

Badr, M. H. (2003) Studying the chemical characteristics of humic acids extracted from soil and stable manure fuel as a new rich source of humic acid and testing its effects on seed germination. Bulletin of Faculty of Science, Al-Azhar University 14,129-140.

Baker H., Khalili F., (2004). Analysis of the removal of lead(II) from aqueous solutions by adsorption onto insolubilized humic acid: temperature and $\mathrm{pH}$ dependence, Anal. Chim. Acta 516, 179-186.

Duncan D.A., Bodle W.W., Banejerd D.P., (1981). Energy from biomass and waste. 5th Symposium, Papers: Institute of Gas Technology, Chicago, pp. 917.

Eladia M. PeÒa-MÈndez, Josef Havel , Jiř̀̀ Patočka, (2005). Humic substances ñ compounds of still unknown structure: applications in agriculture, industry, environment, and biomedicine J. Appl. Biomed. 3, 13-24.

Engebretson, R.R., and von Wandruszka, R. (1998).Micro-Organization in Dissolved Humic Acids. Environ. Sci. Technol. 28, 1934-1941.

Hagenhoff „K., Dong, Jingfeng., Chowdhry, B. Z., Leharne, S.A.( 2010). Aqueous Solution of Anionic Surfactants Mixed with Soils Show a Synergistic Reduction in Surface Tension Water Air Soil Pollut209:3-13

Kerek, M., R.A. Drijber, and R.E. Gaussoin. (2003). Labile soil organic matter as a potential nitrogen source in golf greens. Soil Biol. \& Biochem. 35,1643-1649.

Khalaf M., Kohl S.D., Klumpp E., Rice J.A., Tombacz E., (2003).Comparison of sorption domains in molecular weight fractions of a soil humic acid using solid-state F-19 NMR, Environ. Sci. Technol. $37,2855-2860$

Klavins, M ., Purmalis, O.(2013). Surface activity of humic acids depending on their origin and humification degree, Proc. Latvian Acad. Sci., Section B, 67( 6),493-499.

Tennouga, L., Mansri, A., Medjahed, K., Chetouani, A., Warad, I.( 2015) .The micelle formation of cationic and anionic surfactants in aqueous medium: Determination of CMC and thermodynamic parameters at different temperatures. J. Mater. Environ. Sci. 6 (10) 2711-2716.

Mahmood, M. E., Al-Koofee, D.A.F. (2013). Effect of Temperature Changes on Critical Micelle Concentration for Tween Series Surfactant, Global Journal of Science Frontier Research Chemistry,13(4).

Madejón E, López R, Murillo JM, Cabrera F. . (2001). Agricultural use of three (sugarbeet) vinasse composts: effect on crops 
and chemical properties of a Cambisol soil in the Guadalquivir river valley (SW Spain) Agric Ecosyst Environ; 84,55-65.

Myers, D. (1988). Surfactant science and technology. Wiley Online Library.

Quadri G., Chen X., Jawitz J. W., Tambone F., Genevini P., Faoro F. and Adani F. (2008). Biobased surfactant-likemolecules from organic wastes: The effect of waste composition and composting process on surfactant properties and on the to solubilize tetracloroethene (PCE). Environ. Sci. Technol. 42, 2618-2623.

Patil, S.R., Mukaiyama, T., Rakshit, A.K., Disper, J. (2003). Interfacial, Thermodynamic, and Performance Properties of $\alpha$-Sulfonato Myristic Acid Methyl EsterHexaoxyethylene Monododecyl Ether Mixed Surfactants Sci. Technol. 24 .659.

Pertusatti J. and Prado A.(2007). Buffer capacity of humic acid: Thermodynamic approach, J. Colloid Interf. Sci. 314, 484-489.

Rehman N., Ullah H., Alam S., Jan, Khan S. and Tariq M. (2017). Surface and thermodynamic study of micellization of non ionic surfactant/ diblock copolymer system as revealed by surface tension and conductivity, JMES, 8(4) 1161-1167.

Rosen, M. J., Kunjappu, J.T. (2012). surfactants and interfacial phenomena. $4^{\text {th }}$ ed., Hoboken, New Jersey.

Swift R. S. (1996). In Organic matter characterization. Madison WI 1018-1020.

Schmeide K., S. Pompe, M. Bubner, K.H. Heise, G. Bernhard, H. Nitsche, (2000) Uranium (VI) sorption onto phyllite and selected minerals in the presence of humic acid. Radiochim. Acta 88,723-728.

Simpson, A.J., Determining the molecular weight, aggregation, structures and interactions of natural organic matter using diffusion ordered spectroscopy. Magnetic Resonance in Chemistry, 2002. 40 (13) 72-S82.

Terashima M., Fukushima M., Tanaka S., (2004) Influence of $\mathrm{pH}$ on the surface activity of humic acid: micelle-like aggregate formation and interfacial adsorption, Colloids Surf. APhysicochem. Eng. Aspects 247, 77-83.
Thiam A.R., Bremond N., Bibette J. (2012). From Stability to Permeability of Adhesive Emulsion Bilayers. Langmuir. 28, 6291-6298.

Tombácz , E. (1999). Colloidal properties of humic acids and spontaneous changes of their colloidal state under variable solution conditions. Soil Sci. 164, 814-824.

Wandruszka, V .R. ( 2000). Humic acids: Their detergent qualities and potential uses in pollution, Geochem. Trans.,2

Zhang, M. and He, Z. (2004). long-term changes in organic carbon and nutrients of an Ultisol under rice cropping southeast china. Geoderma 118,167-179. 